\title{
Capecitabine in combination with docetaxel and mitomycin $C$ in patients with pre-treated tumours: results of an extended phase-I trial
}

\author{
T Ernst', K Merx', U Gnad-Vogt', N Lukan', M Kripp', B Schultheis', A Hochhaus' and R-D Hofheinz ${ }^{*, 1}$ \\ 'Onkologisches Zentrum, III. Medizinische Klinik, Medizinische Fakultät Mannheim der Universität Heidelberg, Theodor-Kutzer-Ufer I-3, \\ Mannheim 68167, Germany
}

\begin{abstract}
Preclinical data suggest that the anti-tumour activity of capecitabine is enhanced by taxanes and mitomycin $C$ through up-regulation of thymidine phosphorylase (TP). Here, we studied safety and efficacy of the combination of capecitabine with docetaxel and mitomycin C. Two dose levels (DL) were investigated: capecitabine $1000 \mathrm{mg} \mathrm{m}^{-2}$ b.i.d. on days I - I4, docetaxel $40 \mathrm{mg} \mathrm{m}^{-2}$ i.v. day I, mitomycin C 4 or $6 \mathrm{mg} \mathrm{m}^{-2}$ i.v. day I (DL I or II). Cycles were repeated every 3 weeks. The primary aim was to determine the dose-limiting toxicities (DLT) during the first two treatment cycles and the maximum tolerated dose (MTD). A total of 46 patients (pts) refractory to standard therapies were enrolled, of whom the majority had gastrointestinal tumours $(n=40)$. 14 pts had received $\geqslant 3$ lines of prior chemotherapy. At DL I, one out of six pts experienced DLT. At DL II, two out of six pts had DLT (mucositis grade 3). Thus, DL I was determined as MTD. Among a total of 37 pts treated on DL I the following toxicities were observed during cycles 1 and 2 (number of patients with grade I/2/3/4 toxicity; NCl-CTC v. 3.0): anaemia 10/8/3/0, leucocytopenia 4/I I/I/2, thrombocytopenia 0/I/ 2/0, diarrhoea $8 / 1 / 2 / 0$, stomatitis/mucositis 3/3/I/0, nausea/vomiting 10/2/0/0, and hand-foot skin reaction 5/I/I/0. Of 30 pts who received at least two treatment cycles nine achieved complete or partial remissions, six pts achieved minor remissions, and seven pts had stable disease (tumour control rate 73\%). Of note, four out of 10 patients with pancreatic cancer had partial remissions. In conclusion, capecitabine can safely be combined with docetaxel $\left(40 \mathrm{mg} \mathrm{m}^{-2}\right)$ and mitomycin $C\left(4 \mathrm{mg} \mathrm{m}^{-2}\right)$. The established regimen was well tolerated and the preliminary efficacy data in this heavily pre-treated patients population appears to be promising. British Journal of Cancer (2007) 97, I475-1479. doi: I0.1038/sj.bjc.6604067 www.bjcancer.com
\end{abstract}

Published online 13 November 2007

(C) 2007 Cancer Research UK

Keywords: capecitabine; docetaxel; mitomycin; advanced cancer

To date, no standard recommendations for salvage therapies exist for most types of gastrointestinal tumours with the exception of colorectal cancer. A benefit of salvage therapy has clearly been demonstrated in this disease in three clinical trials comparing either irinotecan, panitumumab or cetuximab against best supportive care (Cunningham et al, 1998; Au et al, 2007; van Cutsem et al, 2007). Randomized trials addressing the question of salvage treatment in other gastrointestinal cancers have not been published so far, and the question how to treat patients with gastrointestinal cancer once beyond the control of first line therapy is unanswered.

Capecitabine, an orally administered fluoropyrimidine carbamate produces high concentrations of 5-fluorouracil (5-FU) preferentially in tumour tissue. It is converted to its active metabolite 5-FU through a three-step enzymatic process, the final step of which is mediated by the enzyme thymidine phosphorylase (TP) (Schuller et al, 2000). Among various cytostatics studied in combination with capecitabine in human cancer xenografts, the

\footnotetext{
*Correspondence: Dr R-D Hofheinz;

E-Mail: ralf.hofheinz@med3.ma.uni-heidelberg.de

Received 23 July 2007; revised 29 September 2007; accepted 9 October 2007; published online 13 November 2007
}

taxanes and mitomycin $\mathrm{C}$ had the highest potential of increasing the activity of intratumoural TP (Sawada et al, 1998). Thus, in an attempt to increase the cytotoxic potential of capecitabine, several studies were conducted using capecitabine in combination with one of these TP inductors.

In a previous trial we established a combination regimen of capecitabine and mitomycin $\mathrm{C}$ which showed a favourable safety profile and promising activity in patients with pre-treated gastrointestinal cancer (Hofheinz et al, 2004). Phase-II studies have confirmed the encouraging activity of this combination with an acceptable toxicity profile in patients with pre-treated (Chong et al, 2005) or chemotherapy-naive (Rao et al, 2004) advanced colorectal cancer. In a randomised phase-II trial comparing mitomycin $\mathrm{C}$ with either capecitabine or gemcitabine in patients with advanced biliary tract cancer, the mitomycin $\mathrm{C}$ plus capecitabine combination was superior in terms of response rate, progression-free survival and overall survival (Kornek et al, 2004). Capecitabine plus mitomycin $\mathrm{C}$ has been demonstrated to be an effective and well tolerated treatment option even in advanced breast cancer patients resistant to anthracyclines and taxanes (Maisano et al, 2005; Massacesi et al, 2006).

The clinical impact of up-regulating TP has further been demonstrated for capecitabine in combination with docetaxel in a variety of human malignancies in phase-I studies (Pronk et al, 
2000; Nadella et al, 2002). In a large phase-III trial the addition of capecitabine to docetaxel resulted in a significant superior survival over single-agent docetaxel in 511 anthracycline-pretreated patients with advanced breast cancer (O'Shaughnessy et al, 2002). This randomised trial revealed that the combination of both drugs provided clear benefits over single-agent docetaxel in terms of objective tumour response rate (42 vs $30 \%$ ), time to progression (median, 6.1 vs 4.2 months), and overall survival (median, 14.5 vs 11.5 months). Furthermore, several phase-II studies on the combination of capecitabine and docetaxel showed activity in patients with metastatic gastric cancer (Chun et al, 2005; Giordano et al, 2006), metastatic oesophageal cancer (Lorenzen et al, 2005), advanced non-small cell lung cancer (Han et al, 2003; Kindwall-Keller et al, 2005), and prostate cancer (Morant et al, 2004; Ferrero et al, 2006).

With the present trial, we sought to establish a three-drug regimen combining capecitabine with both of the preclinical most potent TP inductors, docetaxel and mitomycin C. The primary aim of this clinical trial was to determine the dose-limiting toxicities and the recommended dose of 3-weekly docetaxel and mitomycin $\mathrm{C}$ in combination with standard capecitabine in patients with pretreated tumours.

\section{PATIENTS AND METHODS}

The study protocol was reviewed and approved by the local institutional review board and the study was performed according to the Declaration of Helsinki. All patients provided written informed consent prior to entry into the study.

\section{Eligibility criteria}

Patients with histological confirmed locally advanced or metastatic cancer refractory to or relapsed after at least one palliative chemotherapy regimen were eligible for entry into the study. Additional inclusion criteria comprised: Eastern Cooperative Oncology Group (ECOG) performance status $\leqslant 2$, age $\geqslant 18$ years, life expectancy $\geqslant 3$ months, measurable disease, left ventricular ejection fraction (LVEF) $>50 \%$, cardiac insufficiency NYHA $<2$, adequate bone marrow function (leucocyte count $\geqslant 3000 \mu \mathrm{l}^{-1}$, platelet count $\geqslant 100000 \mu \mathrm{l}^{-1}$ ), and adequate renal (serum creatinine $\leqslant 1.4 \mathrm{mg} \mathrm{dl}^{-1}$ or creatinine clearance $>60 \mathrm{ml} \mathrm{min}^{-1}$ ) and hepatic function (bilirubin $\leqslant 2 \mathrm{mg} \mathrm{dl}^{-1}$ ).

\section{Staging procedures}

Before study admission, all patients underwent a complete history, physical examination, ECG and chest X-rays. Cardiac ultrasonography was carried out to determine the LVEF. A full blood count with differential and serum chemistry was obtained within 14 days prior to the start of treatment. Weekly blood counts were obtained and serum chemistry repeated every third week or whenever clinically indicated. Assessment of LVEF was repeated before the initiation of every other treatment cycle. Computed tomography (CT) scans of the tumour-bearing region were performed within four weeks prior to the start of study treatment. Indicator lesions were assessed every six weeks.

\section{Treatment schedule and dose escalation}

Capecitabine was administered orally within $30 \mathrm{~min}$ after a meal (generally after breakfast and evening meal) at a dose of $1000 \mathrm{mg} \mathrm{m}^{-2}$ b.i.d. on days $1-14$ for all dose levels (DL). At DL I and II docetaxel was administered at a dose of $40 \mathrm{mg} \mathrm{m}^{-2}$ and mitomycin $\mathrm{C}$ was given as an i.v. bolus of $4 \mathrm{mg} \mathrm{m}^{-2}$ (DL I) or $6 \mathrm{mg} \mathrm{m}^{-2}$ (DL II) on day 1 , respectively. At DL III and IV mitomycin $\mathrm{C}$ was scheduled at a fixed dose of $6 \mathrm{mg} \mathrm{m}^{-2}$, and docetaxel was administered at a dose of $50 \mathrm{mg} \mathrm{m}^{-2}$ (DL III) or $60 \mathrm{mg} \mathrm{m}^{-2}$ (DL IV) on day 1 . Cycles were repeated every three weeks. Dexamethasone $8 \mathrm{mg}$ was added intravenously prior to docetaxel/mitomycin $\mathrm{C}$ infusion, and all patients received standard antiemetic prophylaxis with $5-\mathrm{HT}_{3}$ antagonists to avoid any bias relating to gastrointestinal toxicities. A maximum cumulative dose of mitomycin $\mathrm{C}$ was not defined in the study protocol. Nevertheless, a thorough monitoring for parameters of haemolysis/ haemolytic-uraemic syndrome was foreseen by the study protocol.

Dose-limiting toxicities (DLT) during the first two cycles of chemotherapy were defined by the occurrence of one of the following toxicities: grade 4 leucocytopenia/neutropenia or thrombocytopenia, symptomatic thrombocytopenia (haemorrhage), grade 3 or 4 febrile neutropenia or any $\geqslant$ grade 3 non-haematological toxicity except nausea/vomiting. At least three patients were enrolled per dose level, with this number being increased to six if DLT occurred in one patient. Dose escalation was halted if DLT occurred in two or more patients. The maximum tolerated dose (MTD) was defined as the highest dose at which not more than one out of six patients experienced DLT during the first and second course of chemotherapy (i.e. days 1-42). Individual dose escalation was not allowed. In order to adequately determine the safety of this combination, the recruitment of further patients at the MTD was foreseen by the study protocol.

\section{Safety and efficacy analyses}

Adverse events were recorded weekly and graded according to the National Cancer Institute Common Toxicity Criteria (NCI-CTC, version 3.0). In terms of efficacy, complete response (CR), partial response (PR), minor response (MR), stable disease (SD) and progressive disease (PD) were defined according to WHO criteria. Survival was defined as the time between the start of chemotherapy and death, according to the Kaplan-Meier method (Kaplan and Maier, 1958). Progression-free survival was defined as the time between the start of chemotherapy and clinical signs of progressive disease.

\section{Statistical considerations}

For confirmation of the safety of this new regimen the recruitment of further patients at the recommended dose level was foreseen. The frequency of patients experiencing DLT during this expanded study phase was required to be lower than $17 \%$ (i.e. about one out of six of the patients experiencing DLT). With a total of 32 patients, a rate of at least $83 \%$ of the patients experiencing no DLT during cycles one and two can be excluded using Simon's twostage design with a power of $80 \%$ and an $\alpha$-value of $20 \%$ if less than five out of 15 (first stage) or seight out of 32 patients (second stage) experienced DLT.

\section{RESULTS}

\section{Patients' characteristics}

A total of 46 patients were enrolled at our institution between July 2004 and January 2007. All patients were evaluable for safety. Characteristics of enrolled patients are described in Table 1. The majority of patients $(72 \%)$ had an ECOG status $\leqslant 1$. Pancreatic cancer $(28 \%)$, biliary tract cancer $(22 \%)$, colorectal and oesophageal cancer $(15 \%$, each) were the most common malignancies. The liver $(74 \%)$ and lymph nodes $(41 \%)$ were the most common sites of metastases, followed by the lung $(30 \%)$ and bone (17\%). All patients with oesophageal and biliary tract cancers had measurable disease.

Patients had received a median of one line (range 1-6) of prior palliative chemotherapy (14 patients with $\geqslant 3$ lines) and 25 patients (54\%) had received prior 5-FU-based treatment. 
Table I Patient demographics

\begin{tabular}{lc}
\hline Variable & No. of patients (\%) \\
\hline Enrolled & 46 \\
Dose Level I & $37(80)$ \\
Dose Level 2 & $9(20)$ \\
Age (years) & \\
Median & 63 \\
Range & $43-78$ \\
& \\
Gender & \\
Male & $29(63)$ \\
Female & $17(37)$ \\
& \\
Performance status (ECOG) & \\
Median & 1 \\
O & $8(18)$ \\
I & $25(54)$ \\
2 & $13(28)$ \\
Primary tumour site & \\
Pancreatic cancer & $13(28)$ \\
Biliary tract cancer & $10(22)$ \\
Colorectal cancer & $7(15)$ \\
Oesophageal cancer & $7(15)$ \\
Cancer of unknown primary & $3(7)$ \\
Head and neck tumour & $3(7)$ \\
Gastric cancer & $1(2)$ \\
Hepatocellular carcinoma & $1(2)$ \\
Small intestinal cancer & $1(2)$ \\
Prior chemotherapy & \\
I Line & \\
2 Lines & $25(54)$ \\
3 Lines & $7(15)$ \\
5-FU based (i.v. or oral) & $14(31)$ \\
Tumour localisation & $25(54)$ \\
Primary & \\
Liver & \\
Lung & $19(17)$ \\
Lymph nodes & $34(74)$ \\
Bone & $14(30)$ \\
Peritoneal carcinomatosis & \\
\hline & \\
& \\
\hline
\end{tabular}

\section{Determination of the MTD and dose intensity}

One of the first six patients at DL I had DLT (diarrhoea grade 3) and consequently DL II was opened. At DL II nine patients were included. Of these, two patients were not evaluable due to rapid clinical deterioration. Another patient had a non-toxicity-related treatment break of 2,5 weeks between the first and second treatment cycle and was not amenable for the determination of DLT. Two out of the first six evaluable patients included at DL II had DLT (mucositis grade 3) and three patients had leucocytopenia grade 3. Thus, DL I was determined as MTD, and as the recommended dose level for the recruitment of further patients.

Thirty-seven patients were enrolled at dose level I. These patients received a total of 157 cycles (median 4, range 1-13) of chemotherapy. In all, only five patients treated at this DL had DLT during the first four cycles: leucocytopenia grade 4 with mucositis grade 3 , leucocytopenia grade 4 , mucositis grade 3 , hand-foot syndrome grade 3 with diarrhoea grade 3 , diarrhoea grade $3, n=1$ each. Eight patients stopped treatment after cycle one for the following reasons: DLT (leucocytopenia grade $4, n=1$; hand-foot syndrome grade 3 with diarrhoea grade $3, n=1$ ), worsening of general condition $(n=3)$, death due to tumour progression $(n=2)$, consent withdrawn $(n=1)$. At dose level I, 29 out of 37 patients received a minimum of two complete cycles. Of these, 20 patients received at least four cycles, and 15 had five or more cycles of therapy.

Nine patients were enrolled at dose level II. These patients received a total of 32 cycles (median $=2$, range $1-14$ ) of chemotherapy. Four patients stopped treatment after one cycle for the following reasons: DLT (mucositis grade 3, $n=2$ ), death due to tumour progression $(n=1)$, worsening of general condition $(n=1)$. Five patients received a minimum of two complete cycles, three of whom received at least four cycles and two patients five or more cycles of therapy.

Dose intensity was calculated for patients treated at the recommended DL I who received at least two complete cycles of chemotherapy (29/37 patients). A median dose intensity of $100 \%$ was reached during cycles one and two for each drug. The relative mean values with respect to cycles one and two were as follows: capecitabine 96\%, mitomycin and docetaxel $100 \%$, respectively. With respect to cycles three and four, the values were as follows (median/mean; $n=20$ patients): capecitabine 100/96, docetaxel 100/98, mitomycin C 100/99.

Postponement of treatment during cycles one and two was required in three patients only with a mean delay of 20 days (range, 14-24 days). Two of the 20 patients receiving a third or fourth cycle of therapy had a treatment delay with a mean delay of 11 days (range, $7-14$ days).

At the recommended dose level $\mathrm{I}$, the mean cumulative dose of mitomycin C was $31 \mathrm{mg}$ (median $28 \mathrm{mg}$; range $6-91 \mathrm{mg}$ ). 13 of 37 patients $(35 \%)$ received more than $36 \mathrm{mg}$ of mitomycin $\mathrm{C}$ and two patients received more than $76 \mathrm{mg}$.

\section{Safety}

Table 2 summarises haematological and non-haematological adverse events during cycles one and two (the time period for the determination of DLT, i.e. days 1-42) and cycles three and four. At DL I, a total of 37 patients were evaluable for safety. Anaemia $(n=21)$, leucocytopenia $(n=18)$, fatigue $(n=16)$, nausea/vomiting $(n=12)$ and diarrhoea $(n=11)$ were the most commonly observed adverse events during cycles one and two. Leucocytopenia grade 4 was observed in two patients (5\%). Grade 3 toxicities were recorded in nine patients $(24 \%)$ : anaemia $(n=3)$, thrombocytopenia $(n=2)$, diarrhoea $(n=2)$, leucocytopenia, mucositis, hand-foot skin reaction, fatigue $(n=1$, each).

During cycles three and four ( $n=23$ patients), anaemia $(n=15)$, hand-foot syndrome $(n=10)$ and leucocytopenia $(n=9)$ were the most commonly observed adverse events.

No evidence of mitomycin C-induced haemolytic-uraemic syndrome or pulmonary toxicity was documented.

\section{Anti-tumour activity}

30 patients received at least two cycles of treatment and were evaluable for response analysis. One patient with oesophageal and one with biliary tract cancer achieved a complete remission, respectively. Seven patients achieved partial remissions (pancreatic cancer $n=4$, biliary tract cancer $n=1$, oesophageal cancer $n=1$, head and neck tumour $n=1$ ), six patients achieved minor responses (pancreatic cancer $n=4$, biliary tract cancer $n=1$, oesophageal cancer $n=1$ ), and seven patients had stable disease. Anti-tumour activity is summarized in Table 3. Thus, in total 15/30 (50\%) achieved any kind of response and $7 / 30(23 \%)$ had stable disease adding to a tumour control rate of $73 \%$. Only $8 / 30$ (27\%) had progressive disease after cycle 2 while receiving therapy. Remarkably, all of four patients with colorectal cancer had a progressive disease.

The median progression-free survival for all patients $(n=46)$ was 3.2 months (range, $0.2-15.8+$ ), for patients with any kind of tumour response or stable disease $(n=22) 5.0$ months $(1.7-15.8+)$. The duration of the complete remissions observed were $15.8+$ months (biliary tract cancer) and 5 months (oesophageal cancer). The median survival for all patients was 
Table 2 Haematological and non-haematological adverse events per dose level in cycles I/2 and 3/4 (worst per patient)

No. of patients/NCI-CTC grade

\begin{tabular}{|c|c|c|c|c|c|c|c|c|c|c|c|c|c|c|c|c|}
\hline \multirow[b]{3}{*}{ Cycles } & \multicolumn{8}{|c|}{ Dose level I $(n=37)$} & \multicolumn{8}{|c|}{ Dose level $2(n=9)$} \\
\hline & \multicolumn{2}{|c|}{ Grade I } & \multicolumn{2}{|c|}{ Grade 2} & \multicolumn{2}{|c|}{ Grade 3} & \multicolumn{2}{|c|}{ Grade 4} & \multicolumn{2}{|c|}{ Grade I } & \multicolumn{2}{|c|}{ Grade 2} & \multicolumn{2}{|c|}{ Grade 3} & \multicolumn{2}{|c|}{ Grade 4} \\
\hline & $1 / 2$ & $3 / 4$ & $1 / 2$ & $3 / 4$ & $1 / 2$ & $3 / 4$ & $1 / 2$ & $3 / 4$ & $1 / 2$ & $3 / 4$ & $1 / 2$ & $3 / 4$ & $1 / 2$ & $3 / 4$ & $1 / 2$ & $3 / 4$ \\
\hline Anaemia & 10 & 9 & 8 & 6 & 3 & - & - & - & 4 & 1 & 3 & 2 & - & - & - & - \\
\hline Leucocytopenia & 4 & - & 11 & 7 & 1 & 2 & 2 & - & I & 1 & I & - & 3 & 2 & - & - \\
\hline Thrombocytopenia & - & 2 & 1 & - & 2 & - & - & - & - & - & 1 & - & - & - & - & - \\
\hline Nausea/vomiting & 10 & 4 & 2 & - & - & - & - & - & 3 & - & | & - & - & - & - & - \\
\hline Stomatitis/mucositis & 3 & 3 & 3 & 2 & I & 1 & - & - & - & - & - & - & 2 & - & - & - \\
\hline Diarrhoea & 8 & 2 & I & 3 & 2 & - & - & - & - & I & - & - & - & - & - & - \\
\hline Hand-foot skin reactions & 5 & 7 & I & 3 & I & - & - & - & - & - & - & - & - & - & - & - \\
\hline Alopecia & 5 & 5 & 3 & 2 & - & - & - & - & - & - & 2 & 1 & - & - & - & - \\
\hline Fatigue & || & 3 & 4 & 4 & 1 & - & - & - & 3 & - & - & - & - & - & - & - \\
\hline Polyneuropathy & 7 & 3 & - & - & - & - & - & - & - & - & - & - & - & - & - & - \\
\hline Renal function (creatinine) & 2 & 2 & 2 & I & - & - & - & - & - & - & - & - & - & - & - & - \\
\hline
\end{tabular}

${ }^{a}$ Graded according to the National Cancer Institute Common Toxicity Criteria (version 3.0).

Table 3 Anti-tumour activity in patients who received a minimum of two cycles of chemotherapy

\begin{tabular}{lcccccc}
\hline Tumour type & n & CR & PR & MR & SD & PD \\
\hline Pancreatic cancer & 10 & 0 & 4 & 4 & 2 & 0 \\
Biliary tract cancer & 7 & $\mid$ & $\mid$ & $\mid$ & 2 & 2 \\
Oesophageal cancer & 6 & $\mid$ & $\mid$ & $\mid$ & 2 & 1 \\
Colorectal cancer & 4 & 0 & 0 & 0 & 0 & 4 \\
Others & 3 & 0 & $\mid$ & 0 & 1 & 1
\end{tabular}

$\mathrm{CR}=$ complete remission; $\mathrm{PR}=$ partial remission; $\mathrm{MR}=$ minor remission; $\mathrm{SD}=$ stable disease; $\mathrm{PD}=$ progressive disease.

5.2 months $(0.5-17.1+)$, for patients with tumour response or stable disease 8.2 months $(2.1-17.1+)$, for patients with progressive disease 4.5 months $(2.4-7.2+)$.

\section{DISCUSSION}

Docetaxel as well as mitomycin $\mathrm{C}$ caused an up-regulation of thymidine phosphorylase (TP) activity and an enhancement of capecitabine efficacy in a human xenograft model (Sawada et al, 1998). TP activity is frequently induced in human cancers and its induction protects cells from apoptosis and stimulates angiogenesis (Toi et al, 2005). Since TP-targeting treatment has been developed in parallel with analyses of biological functions and translational research, therapies targeting this enzyme might be a rationale chemotherapeutic approach. Combinations between TP-inducible treatments and TP-targeting therapies seem to have improved response and survival in patients with various types of cancer, e.g. breast cancer (O'Shaughnessy et al, 2002). In the present extended phase-I trial we sought to combine capecitabine, a TP-targeting therapy with the two most potent TP-inducing chemotherapeutics, docetaxel and mitomycin C.

Our current findings indicate that capecitabine $1000 \mathrm{mg} \mathrm{m}^{-2}$ orally b.i.d. on days 1-14 in combination with docetaxel $40 \mathrm{mg} \mathrm{m}^{-2}$ and mitomycin C $4 \mathrm{mg} \mathrm{m}^{-2}$ i.v. day 1 every 3 weeks is both effective and well tolerated. Moreover, this regimen appears to be convenient using i.v. application in 3-weekly intervals. Dose escalation in this phase I study continued only to DL II. Due to two patients suffering DLT (mucositis grade 3 ) at this dose level, DL I was determined as MTD. Haematological adverse events, predominantly anaemia and leucocytopenia were the most commonly observed adverse events in a total of 37 patients treated at the MTD. Twenty patients received at least four cycles of chemotherapy on DL I and the toxicity profile observed during the following cycles was comparable. Only hand-foot skin reaction was more frequent in cycles three and four. The median dose intensity was maintained at $100 \%$ during the first four cycles for each drug. In addition, there were no episodes of haemolytic uraemic syndrome or pulmonary toxicities during the study.

The preliminary activity (response rate $30 \%$, tumour control rate $73 \%$ ) observed in 30 patients evaluable for efficacy appeared to be promising in view of the high number of patients with previous 5-FU exposure and the high rate of heavily pre-treated patients in this study. Of note, these results were obtained with comparably low doses of three-weekly docetaxel $\left(40 \mathrm{mg} \mathrm{m}^{-2}\right)$ and mitomycin $\mathrm{C}\left(4 \mathrm{mg} \mathrm{m}^{-2}\right)$. These findings underline our hypothesis that the clinical activity might even be increased by the combination of two instead of one TP-inductor(s) with the TPtargeting agent capecitabine. Of course, these results need confirmation by phase-II trials in distinct tumour entities.

Three-drug regimens containing capecitabine and mitomycin $\mathrm{C}$ have not been reported, while several three-drug combinations containing capecitabine and docetaxel have been published. Capecitabine, docetaxel and gemcitabine were investigated in a phase-I trial in various solid tumours by Amarantidis and coworkers (Amarantidis et al, 2006). The toxicity in 18 patients was described to be generally mild, and responses were seen in some patients. Thirty-five patients with metastatic pancreatic cancer ( $66 \%$ of them chemotherapy-naïve) received this three-drug regimen in a recently published study (Fine et al, 2008). A comparably high rate of tumour remissions (29\%) was reported, but the rate of grade 3-4 thrombocytopenia (14\%) was higher than in the present trial (5\%). Other investigators added cisplatin in a variety of solid tumours (Fakih et al, 2005), carboplatin in metastatic oesophagogastric cancer (Tsai et al, 2005) or epirubicin in patients with advanced breast cancer (Venturini et al, 2003) to the combination of capecitabine and docetaxel. Particularly the phase-II study of Venturini et al described promising anti-tumour activity in a total of 67 patients with chemotherapy-naïve metastastic breast cancer (response rate $82 \%$ ). Nevertheless, a total of $16 \%$ of the patients had febrile neutropenia.

In conclusion, we have established a three-drug dosing schedule for the combination of capecitabine, docetaxel, and mitomycin $\mathrm{C}$ that is well tolerated and showed encouraging signs of activity especially in patients with pancreatic, biliary tract and oesophageal cancer. This regimen may be considered for patients after failure of pre-treatment but it is also likely to be a valuable, cost-saving and convenient treatment option even in earlier stages of cancer chemotherapy. 


\section{REFERENCES}

Amarantidis K, Houhouli K, Papatheodorou K, Miloussis A, Matthaios D, Chatzaki E, Lyrantzopoulos N, Tsaroucha A, Tentes A, Kakolyris S (2006) A dose escalation study of docetaxel plus capecitabine in combination with gemcitabine in patients with advanced solid tumors. Oncol Res 16: $281-287$

Au H, Karapetis C, Jonker D, O’Callaghan C, Kennecke H, Shapiro J, Tu D, Wierzbicki R, Zalcberg J, Moore M (2007) Quality of life in patients with advanced colorectal cancer treated with cetuximab: Results of the NCIC CTG and AGITG CO.17 trial. Proc Am Soc Clin Oncol 22 (Abstract 4002)

Chong G, Dickson JL, Cunningham D, Norman AR, Rao S, Hill ME, Price TJ, Oates J, Tebbutt N (2005) Capecitabine and mitomycin C as third-line therapy for patients with metastatic colorectal cancer resistant to fluorouracil and irinotecan. Br J Cancer 93: 510-514

Chun JH, Kim HK, Lee JS, Choi JY, Hwangbo B, Lee HG, Park SR, Choi IJ, Kim CG, Ryu KW, Kim YW, Lee JS, Bae JM (2005) Weekly docetaxel in combination with capecitabine in patients with metastatic gastric cancer. Am J Clin Oncol 28: $188-194$

Cunningham D, Pyrhonen S, James RD, Punt CJ, Hickish TF, Heikkila R, Johannesen TB, Starkhammar H, Topham CA, Awad L, Jacques C, Herait P (1998) Randomised trial of irinotecan plus supportive care versus supportive care alone after fluorouracil failure for patients with metastatic colorectal cancer. Lancet 352: $1413-1418$

Fakih MG, Creaven PJ, Ramnath N, Trump D, Javle M, Strychor S, Repinski TV, Zamboni BA, Schwarz JK, French RA, Zamboni WC (2005) Phase I and pharmacokinetic study of weekly docetaxel, cisplatin, and daily capecitabine in patients with advanced solid tumors. Clin Cancer Res 11: $5942-5949$

Ferrero JM, Chamorey E, Oudard S, Dides S, Lesbats G, Cavaglione G, Nouyrigat P, Foa C, Kaphan R (2006) Phase II trial evaluating a docetaxel-capecitabine combination as treatment for hormone-refractory prostate cancer. Cancer 107: 738-745

Fine RL, Fogelman DR, Schreibman SM, Desai M, Sherman W, Strauss J, Guba S, Andrade R, Chabot J (2008) The gemcitabine, docetaxel, and capecitabine (GTX) regimen for metastatic pancreatic cancer: a retrospective analysis. Cancer Chemother Pharmacol 61: 167-175

Giordano KF, Jatoi A, Stella PJ, Foster N, Tschetter LK, Alberts SR, Dakhil SR, Mailliard JA, Flynn PJ, Nikcevich DA (2006) Docetaxel and capecitabine in patients with metastatic adenocarcinoma of the stomach and gastroesophageal junction: a phase II study from the North Central Cancer Treatment Group. Ann Oncol 17: 652-656

Han JY, Lee DH, Kim HY, Hong EK, Yoon SM, Chun JH, Lee HG, Lee SY, Shin EH, Lee JS (2003) A phase II study of weekly docetaxel plus capecitabine for patients with advanced nonsmall cell lung carcinoma. Cancer 98: $1918-1924$

Hofheinz RD, Hartmann JT, Willer A, Oechsle K, Hartung G, Gnad U, Saussele S, Kreil S, Bokemeyer C, Hehlmann R, Hochhaus A (2004) Capecitabine in combination with mitomycin $\mathrm{C}$ in patients with gastrointestinal cancer: results of an extended multicentre phase-I trial. Br J Cancer 91: 834-838

Kaplan EL, Maier P (1958) Non-parametric estimation from incomplete observations. J Am Stat Assoc 53: $457-481$

Kindwall-Keller T, Otterson GA, Young D, Neki A, Criswell T, Nuovo G, Soong R, Diasio R, Villalona-Calero MA (2005) Phase II evaluation of docetaxel-modulated capecitabine in previously treated patients with non-small cell lung cancer. Clin Cancer Res 11: 1870-1876

Kornek GV, Schuell B, Laengle F, Gruenberger T, Penz M, Karall K, Depisch D, Lang F, Scheithauer W (2004) Mitomycin C in combination with capecitabine or biweekly high-dose gemcitabine in patients with advanced biliary tract cancer: a randomised phase II trial. Ann Oncol 15: $478-483$
Lorenzen S, Duyster J, Lersch C, von Delius S, Hennig M, Bredenkamp R, Peschel C, Lordick F (2005) Capecitabine plus docetaxel every 3 weeks in first- and second-line metastatic oesophageal cancer: final results of a phase II trial. Br J Cancer 92: 2129-2133

Maisano R, Caristi N, Mare M, Mafodda A, Carboni R, Montalto E, Iorfida M, Nardi M (2005) Mitomycin C and capecitabine combination (MiXe) in heavily pretreated metastatic breast cancer patients. A dose-finding study. Anticancer Res 25: 4513-4517

Massacesi C, La Cesa A, Marcucci F, Pilone A, Rocchi MB, Zepponi L, Santini D, Tonini G, Burattini L (2006) Capecitabine and mitomycin C is an effective combination for anthracycline- and taxane-resistant metastatic breast cancer. Oncology 70: 294-300

Morant R, Bernhard J, Dietrich D, Gillessen S, Bonomo M, Borner M, Bauer J, Cerny T, Rochlitz C, Wernli M, Gschwend A, Hanselmann S, Hering F, Schmid HP (2004) Capecitabine in hormone-resistant metastatic prostatic carcinoma - a phase II trial. Br J Cancer 90: 1312-1317

Nadella P, Shapiro C, Otterson GA, Hauger M, Erdal S, Kraut E, Clinton S, Shah M, Stanek M, Monk P, Villalona-Calero MA (2002) Pharmacobiologically based scheduling of capecitabine and docetaxel results in antitumor activity in resistant human malignancies. J Clin Oncol 20: $2616-2623$

O’Shaughnessy J, Miles D, Vukelja S, Moiseyenko V, Ayoub JP, Cervantes G, Fumoleau P, Jones S, Lui WY, Mauriac L, Twelves C, Van Hazel G, Verma S, Leonard R (2002) Superior survival with capecitabine plus docetaxel combination therapy in anthracycline-pretreated patients with advanced breast cancer: phase III trial results. J Clin Oncol 20: $2812-2823$

Pronk LC, Vasey P, Sparreboom A, Reigner B, Planting AS, Gordon RJ, Osterwalder B, Verweij J, Twelves C (2000) A phase I and pharmacokinetic study of the combination of capecitabine and docetaxel in patients with advanced solid tumours. Br J Cancer 83: 22-29

Rao S, Cunningham D, Price T, Hill ME, Ross PJ, Tebbutt N, Norman AR, Oates J, Shellito P (2004) Phase II study of capecitabine and mitomycin C as first-line treatment in patients with advanced colorectal cancer. $\mathrm{Br} J$ Cancer 91: 839-843

Sawada N, Ishikawa T, Fukase Y, Nishida M, Yoshikubo T, Ishitsuka H (1998) Induction of thymidine phosphorylase activity and enhancement of capecitabine efficacy by taxol/taxotere in human cancer xenografts. Clin Cancer Res 4: 1013-1019

Schuller J, Cassidy J, Dumont E, Roos B, Durston S, Banken L, Utoh M, Mori K, Weidekamm E, Reigner B (2000) Preferential activation of capecitabine in tumor following oral administration to colorectal cancer patients. Cancer Chemother Pharmacol 45: $291-297$

Toi M, Atiqur RM, Bando H, Chow LW (2005) Thymidine phosphorylase (platelet-derived endothelial-cell growth factor) in cancer biology and treatment. Lancet Oncol 6: 158-166

Tsai JY, Iannitti D, Berkenblit A, Akerman P, Nadeem A, Rathore R, Harrington D, Roye D, Miner T, Barnett JM, Maia C, Stuart K, Safran H (2005) Phase I study of docetaxel, capecitabine, and carboplatin in metastatic esophagogastric cancer. Am J Clin Oncol 28: 329-333

van Cutsem E, Peeters M, Siena S, Humblet Y, Hendlisz A, Neyns B, Canon JL, van Laethem JL, Maurel J, Richardson G, Wolf M, Amado RG (2007) Open-label phase III trial of panitumumab plus best supportive care compared with best supportive care alone in patients with chemotherapy-refractory metastatic colorectal cancer. J Clin Oncol 25: $1658-1664$

Venturini M, Durando A, Garrone O, Colozza MA, Contu A, Stevani I, Genta F, Bighin C, Lambiase A, Del Mastro L (2003) Capecitabine in combination with docetaxel and epirubicin in patients with previously untreated, advanced breast carcinoma. Cancer 97: 1174-1180 